\title{
Fluorosomen werfen Licht auf Ribosomenproduktion
}

\author{
ELKE DEUERLING ${ }^{1}$, RAINER NIKOLAY ${ }^{1,2}$ \\ ${ }^{1}$ LEHRSTUHL FÜR MOLEKULARE MIKROBIOLOGIE, UNIVERSITÄT KONSTANZ \\ ${ }^{2}$ INSTITUT FÜR MEDIZINISCHE PHYSIK UND BIOPHYSIK, CHARITÉ BERLIN
}

\section{Ribosomes facilitate protein biosynthesis in all living cells. They consist} of two subunits composed of protein and RNA constituents. Ribosome biogenesis is insufficiently explored due to its complexity and, at the same time, supposed to be an attractive target for new antimicrobials. For both scientific and pharmaceutical purposes the use of fluorescently labeled ribosomes (fluorosomes) could be a valuable tool.
Bakterielle Ribosomen bestehen aus einer einheit. Die 30S-Untereinheit verfügt über ein kleinen (30S) und einer großen (50S) Unter- ribosomales RNA-Molekül (16S-rRNA), das mit 21 ribosomalen Proteinen (rProteinen) interagiert, während die 50S-Untereinheit

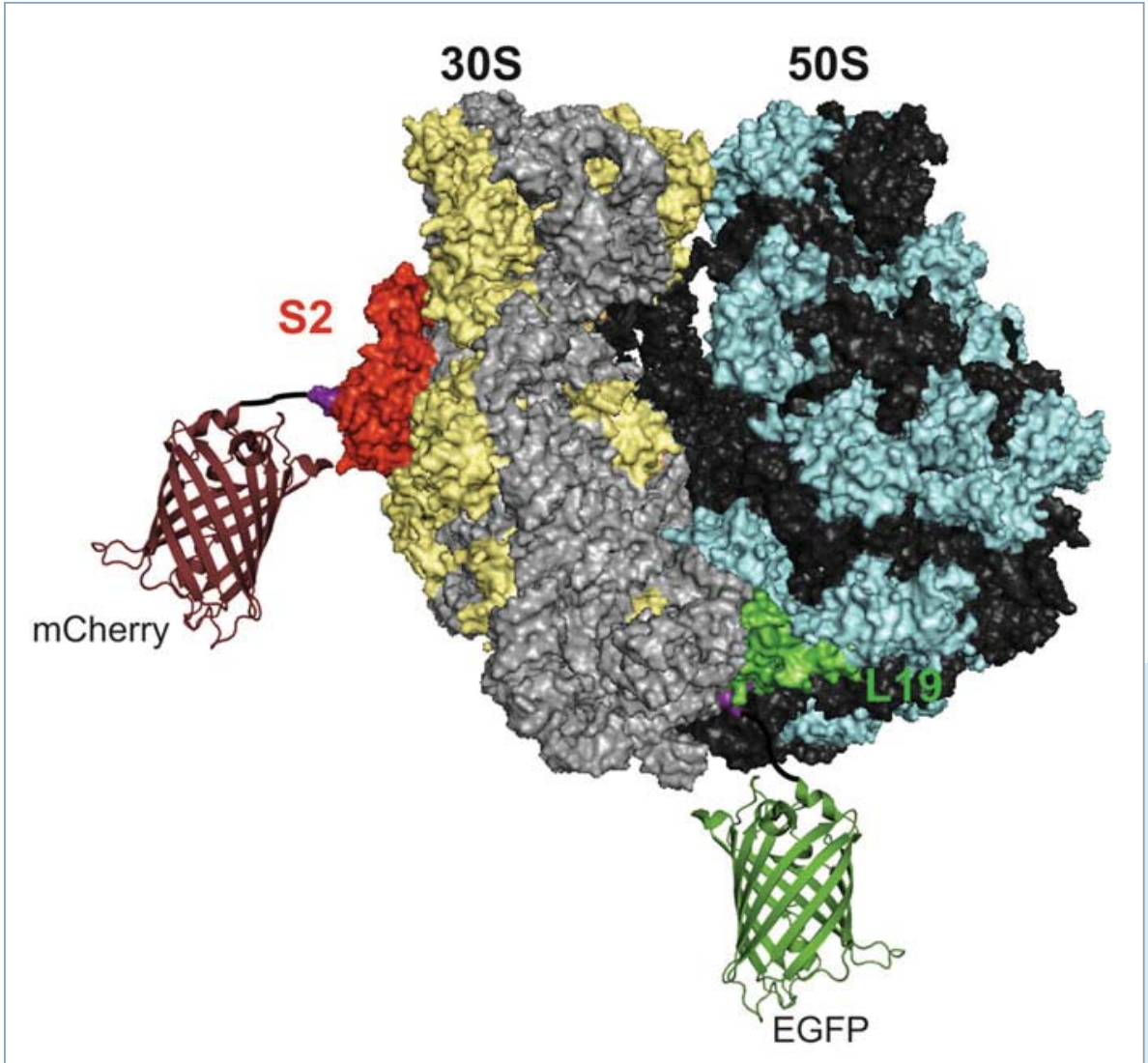

zwei RNA-Moleküle (23S- und 5S-rRNA) und 33 rProteine enthält [1]. Die Entstehung der Ribosomen (Ribosomenbiogenese) in der Zelle beginnt mit der Transkription der rRNA. Bereits mit der naszierenden rRNA treten erste rProteine in Wechselwirkung. Man unterscheidet hierbei früh, mittel und spät interagierende rProteine nach der Reihenfolge, in der ribosomale Untereinheiten durch fortgesetzte Proteinanlagerung reifen [1]. Durch in vitro-Experimente mit gereinigten ribosomalen Komponenten konnten Wissenschaftler regelrechte Baupläne erarbeiten, die ribosomalen Assemblierungskarten [2, 3]. Während ribosomale Untereinheiten in vitro bei hoher Salzkonzentration und Wärmezufuhr spontan entstehen, sind in der Zelle zahlreiche, als Biogenesefaktoren bezeichnete Enzyme involviert. Viele dieser Faktoren sind vermutlich noch unbekannt. Trotz ihrer Komplexität dauert die Entstehung ribosomaler Untereinheiten nur wenige Minuten [4]. Hierdurch wird die Menge frei in der Zelle diffundierender rProteine gering gehalten $(<2 \%)$ [5].

\section{Attraktives Ziel für neue Wirkstoffe}

Die Ribosomenbiogenese bietet wegen ihrer Komplexität viele Angriffspunkte für neuartige antibakterielle Wirkstoffe. Neben Biogenesefaktoren sind auch rProteine ein potenzielles Ziel. Die Blockierung der Interaktion essenzieller rProteine mit der rRNA hat für Bakterien letale Konsequenzen. Wirkstoffkandidaten wären zudem kleine Mole-

4 Abb. 1: Schematische Darstellung des fluoreszenzmarkierten 70S-Ribosoms. 30S-Untereinheit mit rRNA (hellgrau) und S-Proteinen (gelb); S2 (rot), der C-Terminus (violett) ist über einen Aminosäure-Linker (schwarz) verbunden mit dem rot fluoreszierenden Protein mCherry. 50S-Untereinheit mit rRNA (dunkelgrau) und L-Proteinen (cyan); L19 (grün), der C-Terminus (violett) ist über einen Aminosäure-Linker (schwarz) verbunden mit dem grün fluoreszierenden Protein EGFP. 
küle, welche die Bildung oder Auflösung bestimmter Tertiärstrukturen der rRNA behindern. Die rRNA gilt als besonders interessanter Angriffspunkt, weil sie in Bakterien von Genen codiert wird, die in mehrfacher Kopienzahl vorliegen (sieben bei Escherichia coli). Das Risiko einer Resistenzbildung wäre somit stark vermindert. Während sich zytosolische eukaryotische und prokaryotische Ribosomen in Aufbau und Funktionsweise im Detail unterscheiden, und damit auch in ihrer Empfindlichkeit gegenüber Antibiotika, sind mitochondriale und bakterielle Ribosomen einander funktionell sehr ähnlich. Das erklärt die teils starken Nebenwirkungen vieler Antibiotika auf mitochondriale Ribosomen. Allerdings unterscheiden sich die Abläufe der Biogenese von mitochondrialen gegenüber denen von bakteriellen Ribosomen erheblich [6]. Damit hätten potenzielle Inhibitoren der bakteriellen Ribosomenbiogenese wahrscheinlich keine oder nur geringe Nebenwirkungen auf mitochondriale Ribosomen.

\section{Genetisch veränderte Bakterien mit fluoreszierenden Ribosomen}

Für die Identifizierung möglicher Inhibitoren der Ribosomenbiogenese wird ein solides Screeningverfahren benötigt. Als erster Schritt hierfür muss ein geeigneter Bakterien-Reporterstamm etabliert werden. Auf der Grundlage der eingangs genannten Fakten und einer Reihe zusätzlicher Erwägungen haben wir Anforderungen an einen bakteriellen Reporterstamm definiert, um Biogenesedefekte mithilfe fluoreszenzbasierter Ausleseverfahren sichtbar machen zu können: (1) Da freie, nicht ribosomengebundene rProteine kaum vorliegen, entspricht die Menge eines ausgewählten rProteins der Menge der zugehörigen Untereinheit. (2) Die Fluoreszenzmarkierung eines spät interagierenden rProteins gewährleistet, dass möglichst nur vollständig gereifte, intakte Ribosomen markiert werden. (3) Die Markierung von rProteinen erfolgt durch Fusionierung mit fluoreszierenden Proteinen. Dazu wird die codierende Sequenz des rProtein-Gens mit dem offenen Leseraster eines fluoreszierenden Proteins verknüpft [7]. (4) Die ausgewählten rProteine sollten über einen Oberflächen-exponierten Terminus verfügen, um sie ohne Funktionsverlust mit fluoreszierenden Proteinen fusionieren zu können.
Basierend auf diesen Überlegungen wählten wir die rProteine S2 und L19 für die Fusionierung mit fluoreszierenden Proteinen aus. Mithilfe von Standardmethoden der Bakteriengenetik erzeugten wir durch chromosomalen Gen-Knock-in Ribosomen, deren Untereinheiten unterschiedlich markiert sind: Fusion von S2 mit mCherry färbt die kleine Ribosomenuntereinheit rot, Verknüpfung von L19 mit EGFP (enhanced grün fluoreszierendes Protein) die große Untereinheit grün (Abb. 1, [8]). Bakterien mit derartig veränderten Ribosomen - Fluorosomen haben eine ähnliche Zellteilungsrate und Stressresistenz wie der Wildtyp. Das deutet darauf hin, dass die markierten Ribosomen des Reporterstamms Wildtyp-artig funktionieren. Damit kann ausgeschlossen werden, dass der Reporterstamm bereits Ribosomendefekte aufweist.

\section{Funktionsnachweis durch künstliche Ribosomendefekte}

Zur Validierung des Reporterstamms als zuverlässigen Detektor einer inhibierten Ribosomenbiogenese entwickelten wir zusätzliche Varianten dieses Bakterienstamms. Laut Fachliteratur rufen Mutationen in bestimmten Genen der kleinen und großen Untereinheit untereinheitenspezifische Defekte hervor $[9,10]$. Dementsprechend konstruierten wir Reporterstämme, bei denen entweder ein Gen der kleinen (rpsQ, codiert S17) oder der großen Untereinheit ( $r p l C$, codiert L3) inaktiviert werden kann. Tatsächlich führt die Entfernung des rpsQ-Gens zu einem Defekt der kleinen und von $r p l C$ zu einem Defekt in der großen Untereinheit (Abb. 2). Um die Intaktheit von Ribosomen zu prüfen, wird Zellextrakt durch einen Dichtegradienten zentrifugiert. Dies ermöglicht die Trennung der einzelnen Untereinheiten (30S und 50S), 70S-Ribosomen und Polysomen (mehrere Ribosomen, die eine mRNA translatieren) sowie die kontinuierliche Darstellung der Messergebnisse in einem Diagramm: Gemessen wird die Absorption bei einer Wellenlänge von 260 Nanometern, wodurch die in den Ribosomen enthaltene Nukleinsäure erfasst wird. Gleichzeitig kann die Fluoreszenz im roten und grünen Bereich detektiert und den ribosomalen Untereinheiten zugeordnet werden. Die verschiedenen Signale können kombiniert dargestellt werden, da es sich um unterschiedliche Ausleseverfahren derselben 


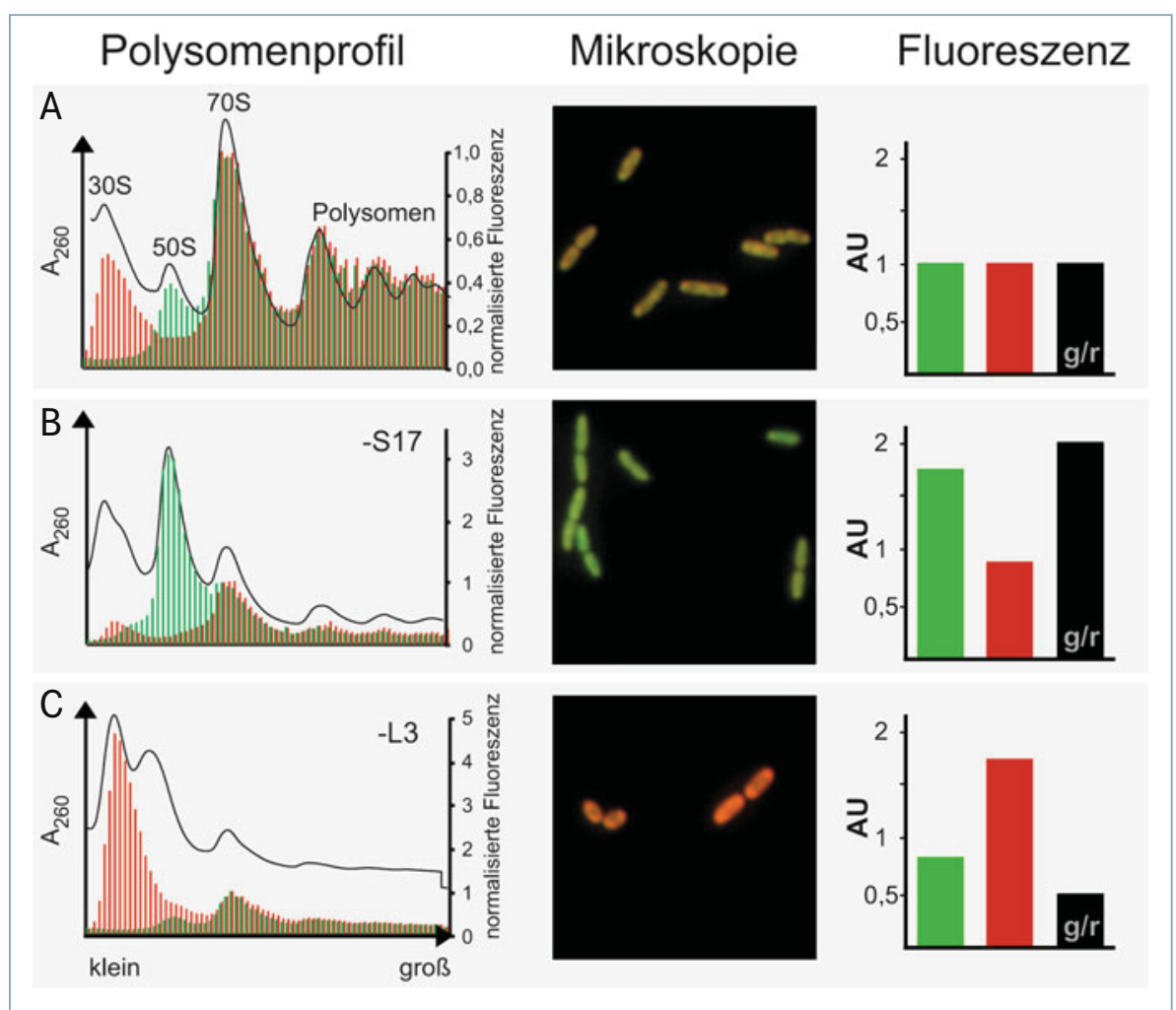

$\Delta$ Abb. 2: Fluorometrische Untersuchung von Reporterstamm und Mutanten. A, Reporterstamm. B, Mutant mit Depletion von S17 (-S17). C, Mutant mit Depletion von L3 (-L3). Jeweils links: Polysomenprofil (schwarze Linie) mit 30S-, 50S-Untereinheiten, 70S-Ribosomen und Polysomen; mCherry-markierte 30S-Untereinheiten (rote Säulen) und EGFP-markierte 50S-Untereinheiten (grüne Säulen). Während sich Polysomen- und Fluoreszenzprofile des Reporterstamms decken (A), deuten die Differenzen bei den Mutanten auf einen 30S- (B) oder einen 50S-Defekt (C) hin. Mitte: fluoreszenzmikroskopische Aufnahmen einzelner Bakterienzellen. Rechts: Darstellung von grüner und roter Fluoreszenz sowie des Quotienten aus beiden Fluoreszenzintensitäten ( $\mathrm{g} / \mathrm{r}$ ). AU: arbitrary units (willkürliche Einheiten) (nach [8]).

\section{Danksagung}

Die Manuskripterstellung wurde unterstützt von Klaus Karp (www.wortkarp.com). Die wissenschaftlichen Arbeiten von Elke Deuerling wurden mit Mitteln der DFG (SFB969/A01) und des Human Frontier Science Program (HFSP) gefördert.

\section{Literatur}

[1] Shajani Z, Sykes MT, Williamson JR (2011) Assembly of bacterial ribosomes. Annu Rev Biochem 80:501-526 [2] Nierhaus KH, Dohme F (1974) Total reconstitution of functionally active $50 \mathrm{~S}$ ribosomal subunits from Escherichia coli. Proc Natl Acad Sci USA 71:4713-4717

[3] Traub P, Nomura P (1968) Structure and function of E. coli ribosomes. V. Reconstitution of functionally active $30 \mathrm{~S}$ ribosomal particles from RNA and proteins. Proc Natl Acad Sci USA 59:777-784

[4] Lindahl L (1975) Intermediates and time kinetics of the in vivo assembly of Escherichia coli ribosomes. J Mol Biol 92:15-37

[5] Marvaldi J, Pichon J, Delaage M et al. (1974) Individual ribosomal protein pool size and turnover rate in Escherichia coli. J Mol Biol 84:83-96

[6] Bogenhagen DF, Martin DW, Koller A (2014) Initial steps in RNA processing and ribosome assembly occur at mitochondrial DNA nucleoids. Cell Metab 19:618-629

[7] Shaner NC, Steinbach PA, Tsien RY (2005) A guide to choosing fluorescent proteins. Nat Methods 2:905-909 [8] Nikolay R, Schloemer R, Schmidt S et al. (2014) Validation of a fluorescence-based screening concept to identify ribosome assembly defects in Escherichia coli. Nucleic Acids Res 42:e100

[9] Lhoest J, Colson C (1981) Cold-sensitive ribosome assembly in an Escherichia coli mutant lacking a single methyl group in ribosomal protein L3. Eur J Biochem 121:33-37 [10] Herzog A, Yaguchi M, Cabezón T et al. (1979) A missense mutation in the gene coding for ribosomal protein S17 (rpsQ) leading to ribosomal assembly defectivity in Escherichia coli. Mol Gen Genet 171:15-22

Korrespondenzadresse:

Prof. Dr. Elke Deuerling

Lehrstuhl für Molekulare Mikrobiologie

Universität Konstanz

Universitätsstraße 10

D-78457 Konstanz

Tel.: 07531-882647

Fax: 07531-884036

Elke.Deuerling@uni-konstanz.de

www.biologie.uni-konstanz.de/deuerling schließen lassen. Die Untersuchung des Reporterstamms und der beiden Mutanten im Fluoreszenzmikroskop führt zu einem sehr ähnlichen Ergebnis. Während der Reporterstamm gelblich erscheint, dominiert bei den Mutanten - aufgrund ihrer untereinheitenspezifischen Defekte - entweder grün oder rot. Diese Untersuchungsergebnisse bestätigen die Funktionsfähigkeit der vorgestellten auf Fluorosomen basierten Methode zum Nachweis von Störungen der Ribosomenbiogenese.

\section{Neue antibakterielle Wirkstoffe}

Der beschriebene Reporterstamm ermöglicht ein fluoreszenzbasiertes in vivo-Screening und könnte damit interessante Leitsubstanzen liefern, die durch Optimierung und 